\title{
Performance Analysis of Multiscale Entropy for the Assessment of ECG Signal Quality
}

\author{
Yatao Zhang, ${ }^{1,2}$ Shoushui Wei, ${ }^{1}$ Yutao Long, ${ }^{1}$ and Chengyu Liu' \\ ${ }^{1}$ School of Control Science and Engineering, Shandong University, Jinan 250061, China \\ ${ }^{2}$ School of Mechanical, Electrical \& Information Engineering, Shandong University, Weihai 264209, China \\ Correspondence should be addressed to Shoushui Wei; sswei@sdu.edu.cn
}

Received 6 January 2015; Revised 9 April 2015; Accepted 15 April 2015

Academic Editor: Mohamad Sawan

Copyright (C) 2015 Yatao Zhang et al. This is an open access article distributed under the Creative Commons Attribution License, which permits unrestricted use, distribution, and reproduction in any medium, provided the original work is properly cited.

\begin{abstract}
This study explored the performance of multiscale entropy (MSE) for the assessment of mobile ECG signal quality, aiming to provide a reasonable application guideline. Firstly, the MSE for the typical noises, that is, high frequency (HF) noise, low frequency (LF) noise, and power-line (PL) noise, was analyzed. The sensitivity of MSE to the signal to noise ratio (SNR) of the synthetic artificial ECG plus different noises was further investigated. The results showed that the MSE values could reflect content level of various noises contained in the ECG signals. For the synthetic ECG plus LF noise, the MSE was sensitive to SNR within higher range of scale factor. However, for the synthetic ECG plus HF noise, the MSE was sensitive to SNR within lower range of scale factor. Thus, a recommended scale factor range within 5 to 10 was given. Finally, the results were verified on the real ECG signals, which were derived from MIT-BIH Arrhythmia Database and Noise Stress Test Database. In all, MSE could effectively assess the noise level on the real ECG signals, and this study provided a valuable reference for applying MSE method to the practical signal quality assessment of mobile ECG.
\end{abstract}

\section{Introduction}

ECGs collected via mobile phone are easily contaminated by system noises, body movement, and circumstance disturbing so that the corrupted data could lead to false alarms and misdiagnosis [1]. Thus there is an essential requirement to assess quality of ECG in order to determine whether the ECG can be used for clinical purpose. Recently, ECG quality assessment has been a focus issue [2-8].

ECG waveforms and power spectra are usually in chaos when the ECGs are corrupted badly by noises. So methods currently available employ the metrics mostly based on the characterisation of time or frequency features of the signals. Time based methods aim to identify particular characteristics like RR time interval outliers [4], flat lines, baseline drift, baseline wandering, and steep slopes [5] which can usually compromise the recordings. Frequency based methods can use, for example, the ratio between the low- and the highfrequency content of the signals [6]. A combination of time and frequency methods has also been proposed $[7,8]$. The traditional wave form features and power spectrum are relatively simple metrics for assessing quality of ECG; however it is difficult to further improve classification accuracy. So the proper metrics are required to further reflect the content level of noises contained in ECG. Due to ECG being a nonlinear signal, some nonlinear processing methods are reasonable and feasible.

Multiscale entropy (MSE), a nonlinear analysis measure, has been applied to a variety of biomedical signals [912]. Costa et al. firstly proposed and employed MSE to biomedical signals analysis $[9,10]$. Chung et al. analysed the electroencephalography (EEG) during sleep in patients with Parkinson's disease using MSE [11]. Zhang et al. employed MSE to analyse characterizing different patterns of spontaneous electromyogram (EMG) signals [12] and then got the nonlinear features of these patterns. In these applications, MSE was used for dealing with the signals that were already processed by filtering, correction, and other methods. However ECG quality assessment has to analyse the signals that are without any processing, and the signals are more complex and contain lots of various noises. Besides, the change of the MSE for some common noises contained in ECG and the 
relationship between the MSE and the content level of noises contained in ECG also need to be analysed in ECG quality assessment. This exemplifies the need to further characterise the factors that affect the MSE and particularly in the context of assessing ECG signal quality.

We clarify that, beyond MSE, other types of dynamic entropy have been used to analyze ECG data. For example, the entropy $S$ defined in a time domain termed natural time differs essentially from MSE since it is defined in an entirely different time domain [13]. By quantifying the $S$ fluctuations and using ratios of "shuffled" and "unshuffled" $S$ fluctuations on fixed time scales or ratios on different time scales complexity measures have been introduced that were found of usefulness not only in the ECG analysis but also to discriminate similar looking electric signals emitted from systems of different dynamics.

The aim of this study was to characterise the MSE in ECG signals and assess the effect of typical ECG noises affecting the ECG, with the help of both artificial and real signals.

\section{Materials and Methods}

\subsection{Databases}

2.1.1. Artificial ECG Signals. Four types of artificial signals were considered: ECG representing our actual signal of interest; $0-0.5 \mathrm{~Hz}$ low-frequency (LF) noises as representative of baseline wander (BW) and movement artefacts; $50 \mathrm{~Hz}$ sinusoid as representative of power-line (PL) noise; $50-180 \mathrm{~Hz}$ high-frequency (HF) noises as representative of muscle artefact (MA) and other high-frequency noise. For each of these categories, 50 signals of 50 seconds with a sample rate of $360 \mathrm{~Hz}$ were generated and utilised for the analysis.

The artificial ECG signals were generated utilising the open source ECGSYN software described in McSharry et al. [14]. The ECG signals had a heart rate between $50 \mathrm{bpm}$ and $100 \mathrm{bpm}$ which was randomly chosen for each repeat. Each of the low-frequency signals had a frequency which was randomly chosen from the interval $0-0.5 \mathrm{~Hz}$ and amplitude which was also randomly chosen. The fifty repeats of PL noise signals were generated with a fixed frequency of $50 \mathrm{~Hz}$ sinusoidal signal and random amplitude separately chosen for each repeat. Each of the high-frequency signals had a frequency which was randomly chosen from the interval 50$180 \mathrm{~Hz}$ (based on sample rate of $360 \mathrm{~Hz}$ ) and amplitude which was also randomly chosen.

The study also used three synthetic ECG signals for the analysis of the effect of SNR on the MSE that included the clean artificial ECG plus HF noise, the clean plus LF noise, and the clean plus PL noise. The three types of noisy signals were generated using a SNR in the range from $-20 \mathrm{~dB}$ to $40 \mathrm{~dB}$ in steps of $10 \mathrm{~dB}$. The SNR is defined as

$$
\mathrm{SNR}=10 \times \log _{10}\left(\frac{P_{\text {signal }}}{P_{\text {noise }}}\right),
$$

where $P_{\text {signal }}$ and $P_{\text {noise }}$ denote the power of the clean ECG and the power of the noise, respectively. Figure 1 shows the three types of ECG signals. For each type of signal, SNR was varied from $-20 \mathrm{~dB}$ to $40 \mathrm{~dB}$, with steps of $10 \mathrm{~dB}$. For each SNR level, 50 repeats were produced and the mean value and standard deviation of each type of signal were calculated. For the effect analysis of signal length, SNR of the ECG was set as a constant of $10 \mathrm{~dB}$. For the effect analysis of SNR, the ECG signal length used the recommendation value from the effect analysis of signal length.

2.1.2. Real ECG Signals. The original real ECG signals were taken from the MIT-BIH Arrhythmia Database made available by the Beth Israel Deaconess Medical Center through the PhysioNet website $[15,16]$. Actually, this study had to firstly remove BW from the original real signals because BW contained in the original signals could lead to inaccurate results and then constructed new real ECG signals. This new database contains 48 clean 30-minute 2-channel ambulatory ECG recordings with sample rate of $360 \mathrm{~Hz}$. The MIT-BIH Noise Stress Test Database (NSTDB) [17], also from the PhysioNet website, was also utilised. The NSTDB provides recordings of three typical noise signals which are typically found in ambulatory ECG recordings and include electrode motion artefact (EM), BW, and MA. Because the NSTDB does not include the $50 \mathrm{~Hz}$ PL noise, the study also added this type of noise to the real ECG signals for testing. Figure 2 shows the real ECG and the synthetic ECG plus BW, EM, MA, and PL noise.

2.2. MSE Method. The MSE is obtained from a sample entropy analysis of the signals for different time scale factors. Firstly original time series $\left\{x_{1}, x_{2}, \ldots, x_{L}\right\}$ (length $L$ ) is converted to new multiple time series after a coarse-graining procedure that is performed by averaging the variable data points in nonoverlapping windows by time scale factors. Each element of the new time series $y_{j}^{\tau}$ is computed according to the following equation:

$$
y_{j}^{(\tau)}=\frac{1}{\tau} \sum_{i=(j-1) \tau+1}^{j \tau} x_{i},
$$

where $\tau$ denotes the time scale factor and $1 \leq j \leq L / \tau$. So the new series $\{y(i): 1 \leq i \leq N\}$ is obtained and its length for each scale factor is $N=L / \tau$. For scale 1 , the new time series is consistent with the origin time series.

Then the sample entropy of each new time series is computed. For each new time series, a vector $(N-m+1)$ is constructed as in the following equation:

$$
\begin{aligned}
R_{i}=\{y(i), y(i+1), \ldots, y(i+m-1)\} & \\
& i=1,2, \ldots, N-m+1,
\end{aligned}
$$

where $m$ indicates the embedding dimension.

The distance $d\left[R_{i}, R_{j}\right](i, j=1,2, \ldots, N-m+1, i \neq j)$ between the vectors $R_{i}$ and $R_{j}$ is computed according to the following equation:

$$
\begin{aligned}
d\left[R_{i}, R_{j}\right]=\max [|y(i+k)-y(j+k)|] & \\
& k=0,1, \ldots, m-1 .
\end{aligned}
$$



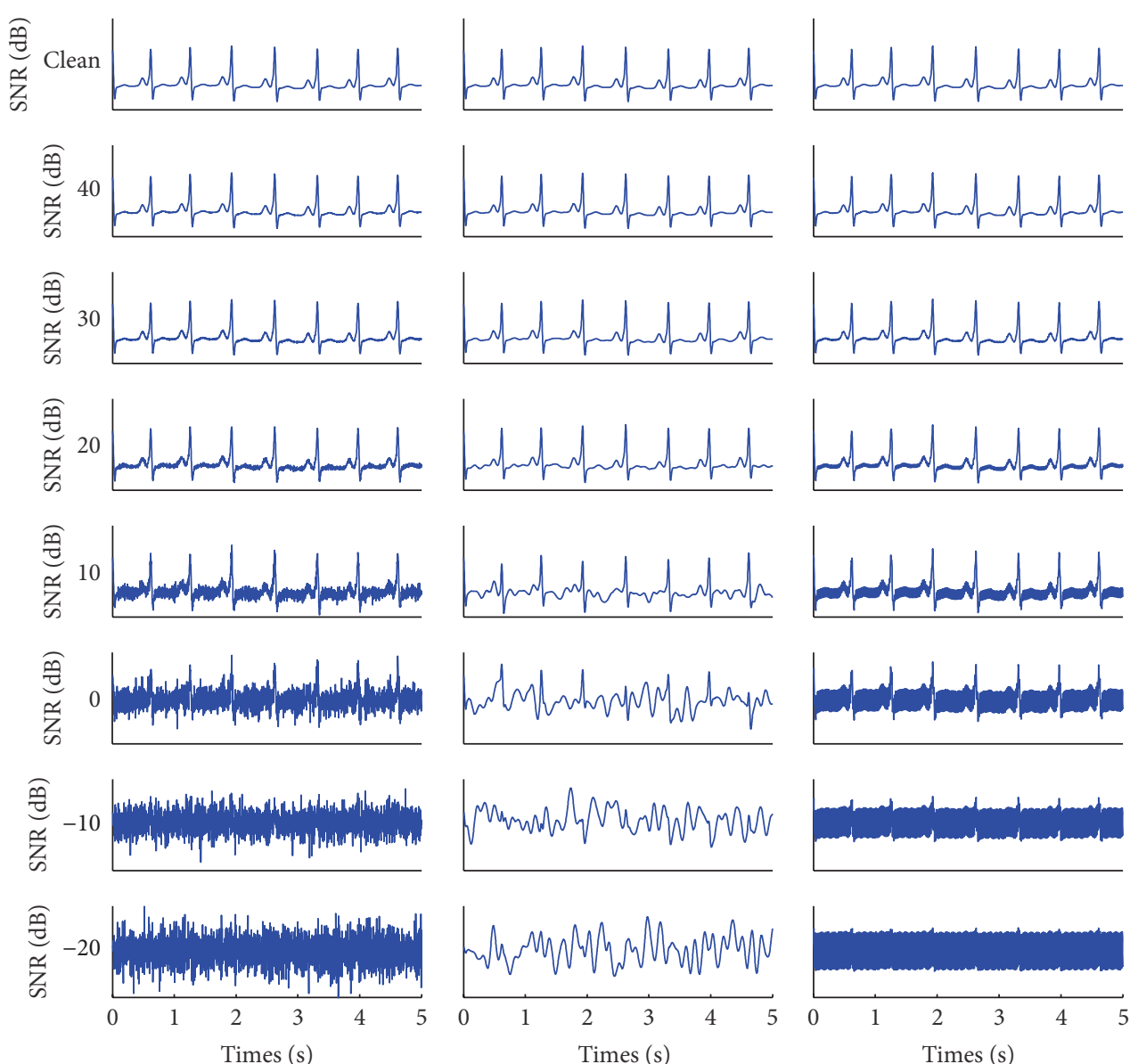

(a)

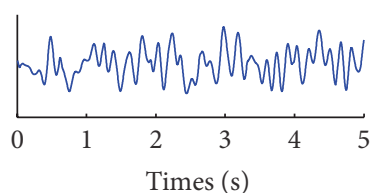

(b)

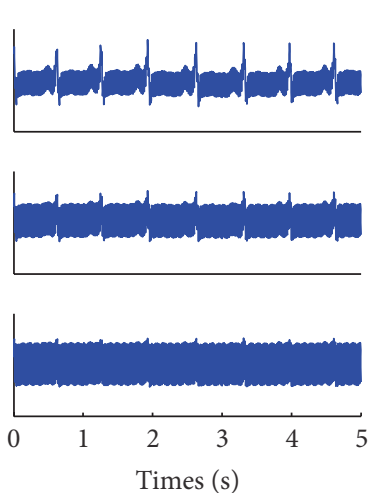

(c)

FIgURE 1: The clean artificial ECG and the clean ECG added noises with different SNRs from $-20 \mathrm{~dB}$ to $40 \mathrm{~dB}$, with a step of $10 \mathrm{~dB}$. (a) The clean ECG and the clean plus HF noise. (b) The clean and the clean plus LF noise. (c) The clean and the clean plus PL noise.

The degree of similarity between $R_{i}^{m}$ and the other vector $R_{j}^{m}$ within tolerance $r$ can be defined as $B_{i}^{m}(r)$, that is, $(N-$ $m)^{-1}$ times the number of the distance $d\left[R_{i}, R_{j}\right]$ within $r$, where $i$ range from 1 to $(N-m+1)$ and $i \neq j$ to exclude self-matches, and then the average degree of similarity for all of $i$ is defined as

$$
B^{m}(r)=\sum \frac{B_{i}^{m}(r)}{(N-m+1)},
$$

where $i=1,2, \ldots, N-m+1$. Similarly, $B^{m+1}(r)$ can also be computed for the embedded dimension of $m+1$. Then, the SampEn is defined as follows:

$$
\operatorname{SampEn}(\tau, m, r)=-\ln \left[\frac{B^{\tau, m+1}(r)}{B^{\tau, m}(r)}\right] .
$$

Finally, the MSE is the set of SampEn on multiscale and defined as

$$
\operatorname{MSE}(s)=\{\tau \mid \operatorname{SampEn}(\tau, m, r)\} .
$$

In this study, the values of $m=2$ and $r=0.15$ for MSE computation were selected, and the scale factor from 1 to 15 was selected.

\subsection{MSE Analysis for the Artificial ECG}

2.3.1. The Relationship between MSE and Signal Types. In order to understand how change of MSE of signal reflected signal types, the values of MSE were firstly characterised separately for each of the four typical artificial signals: HF noise, LF noise, the clean ECG, and PL noise, using 50 repeats for each.

2.3.2. Effect of Signal Length on MSE. The possible effect of the length of the recording on the value of its MSE was assessed by calculating the MSE for the clean plus LF, the clean plus PL, and the clean plus HF noise when SNR was $10 \mathrm{~dB}$ with a length ranging from $5 \mathrm{~s}$ to $85 \mathrm{~s}$, in steps of $5 \mathrm{~s}$, as well as the clean ECG. As before, 50 repeats were considered in each of the four groups. A SNR of $10 \mathrm{~dB}$ was used when generating the noisy ECG signals with HF, PL, and LF noise, respectively.

2.3.3. Effect of the SNR on MSE. The possible effect of the SNR of the signals on value of their MSE was assessed by calculating the MSE for the three types of noisy signals 


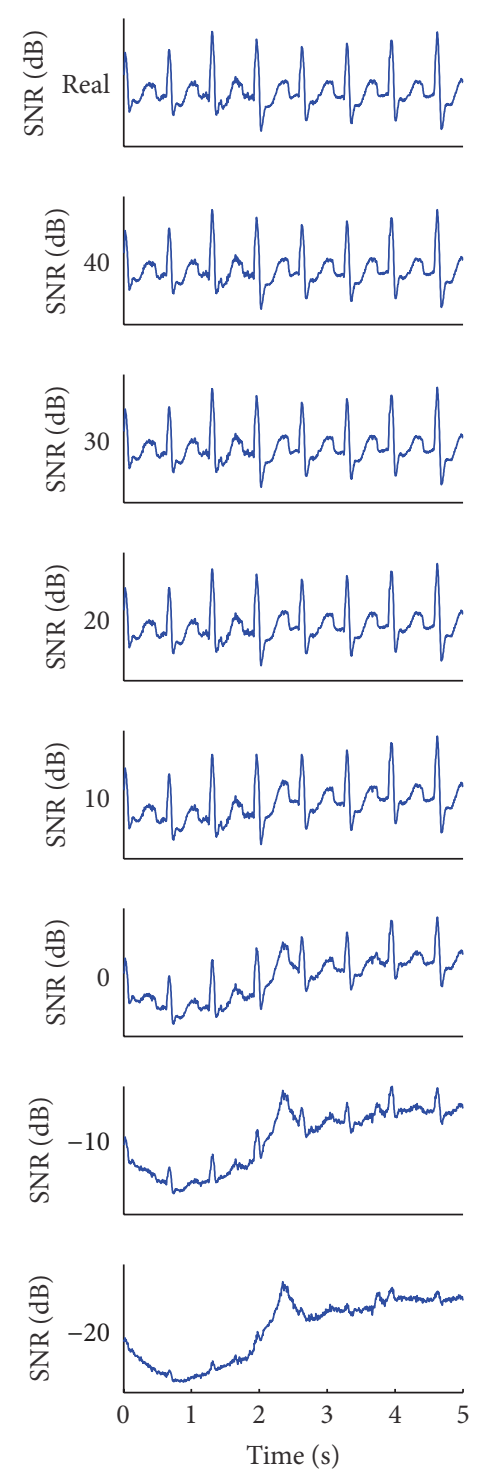

(a)
MAnAmands

InAmandunds

InAmannans

Andmandunds

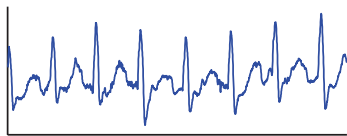

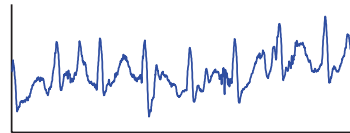
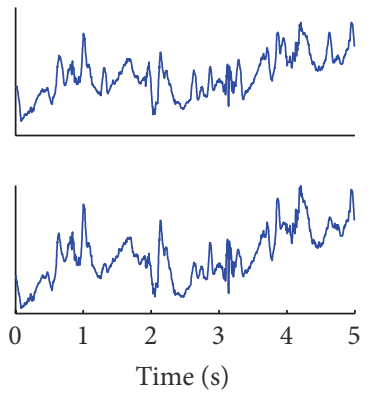

(b)

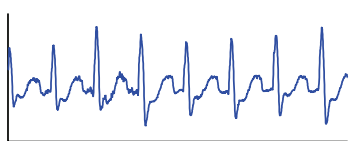

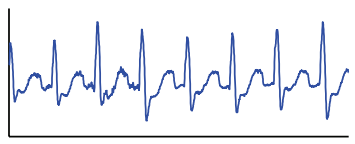

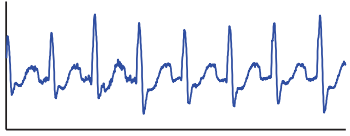

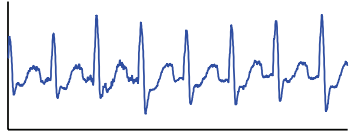

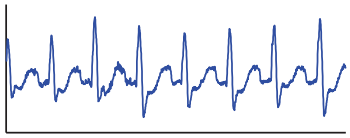

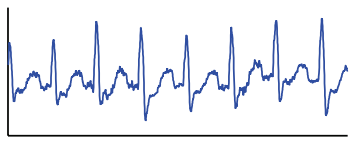
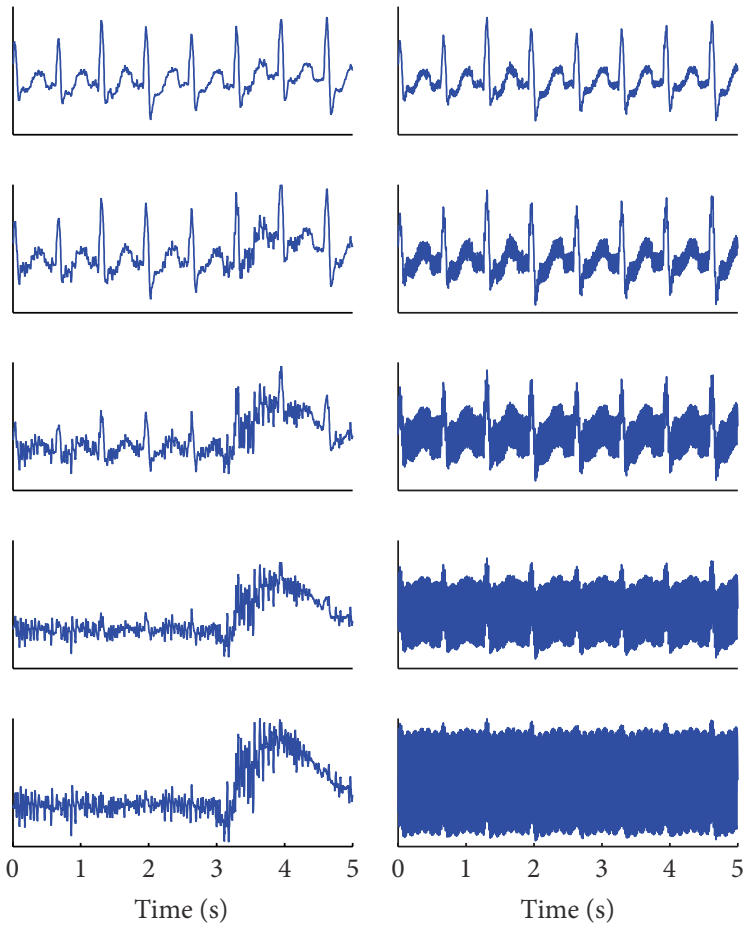

(c)

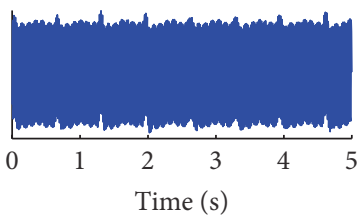

(d)

FIGURE 2: The real ECG and real ECG added noises with different SNRs from $-20 \mathrm{~dB}$ to $40 \mathrm{~dB}$, with a step of $10 \mathrm{~dB}$. (a) The real ECG and the real plus BW. (b) The real ECG and the real plus EM. (c) The real ECG and the real plus MA. (d) The real ECG and the real plus PL noise.

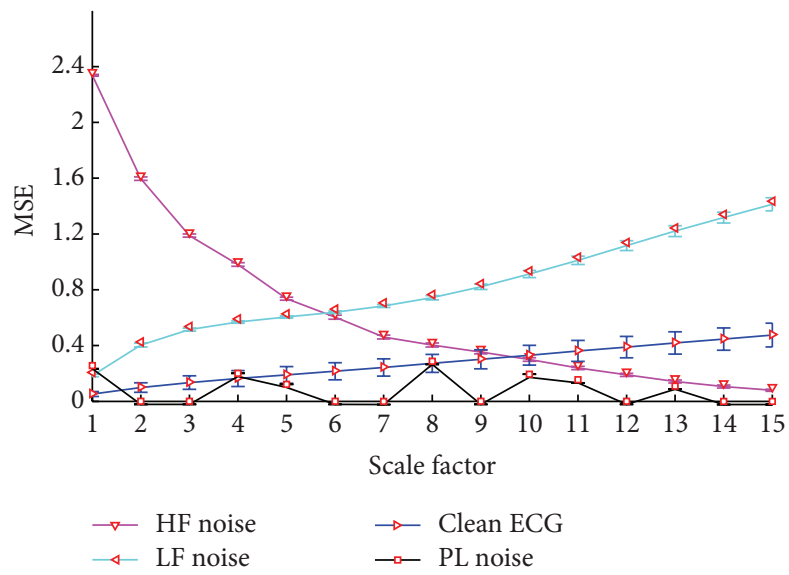

FIGURE 3: MSE of three typical noises and the clean ECG with scale factor from 1 to 15. 


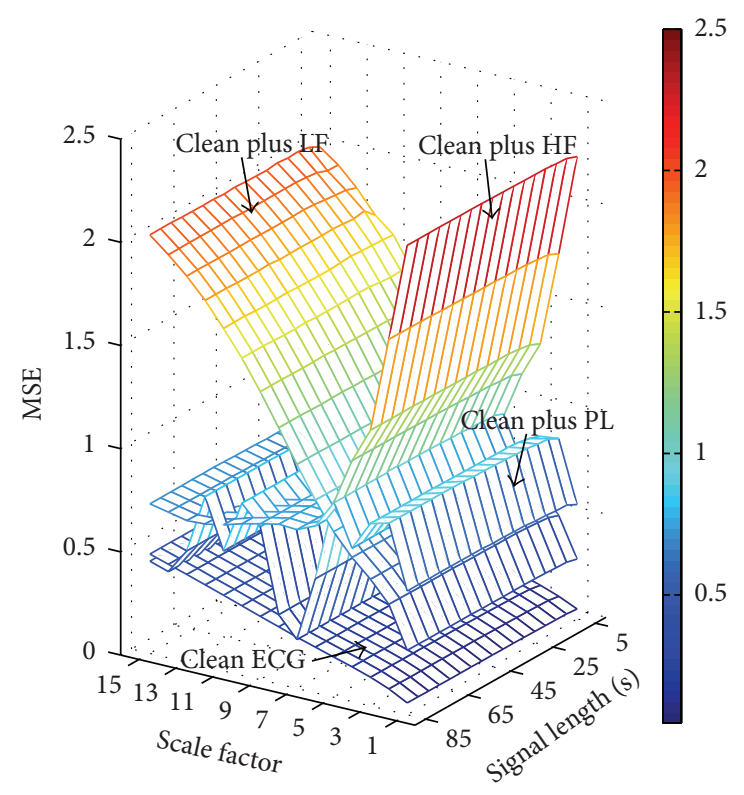

(a)

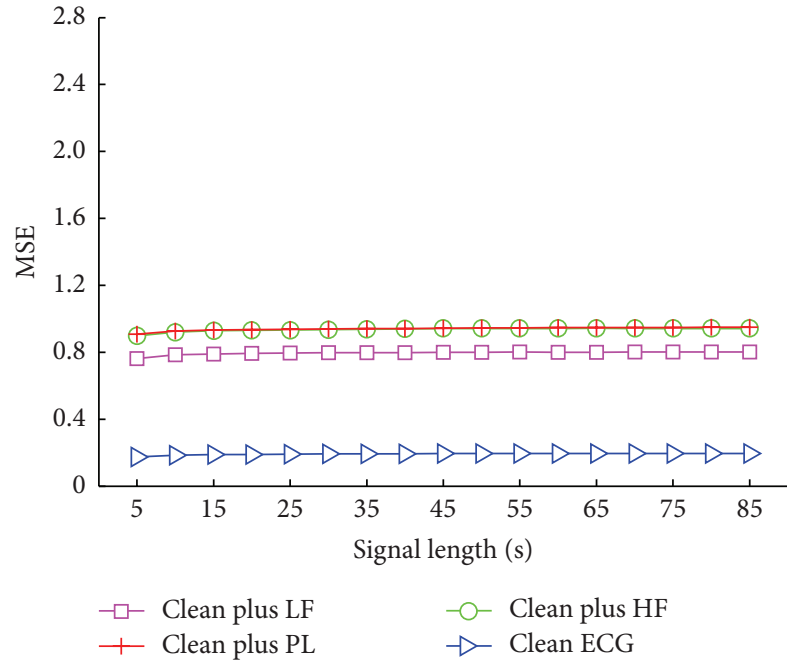

(b)

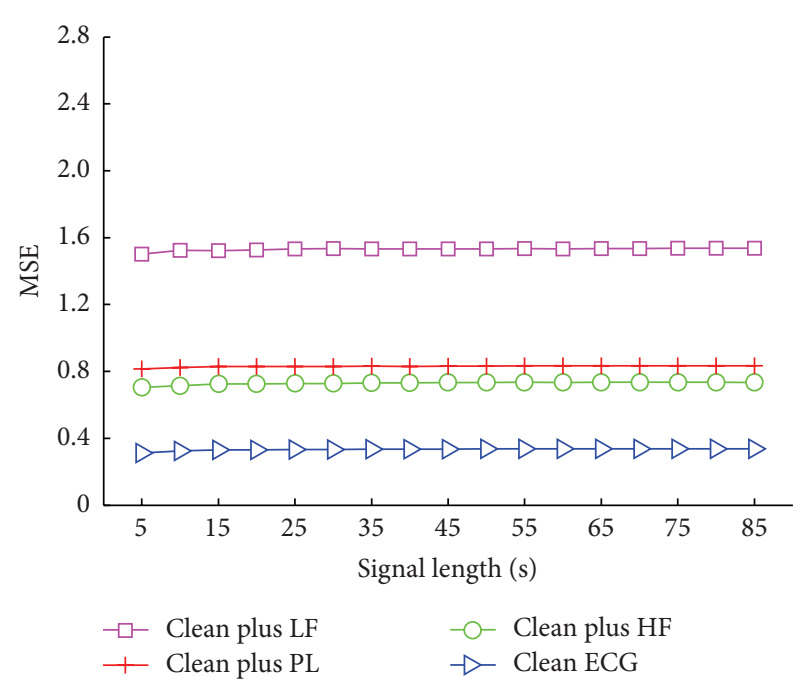

(c)

FIGURE 4: The effect of signal length on MSE for the artificial ECG signals. (a) The 3D view of the effect of signal length on all of the four artificial ECGs. (b) Different lengths and their corresponding MSE with a scale factor of 5. (c) Different lengths and their corresponding MSE with a scale factor of 10 .

described above, generated using a SNR in the range from $-20 \mathrm{~dB}$ to $40 \mathrm{~dB}$ in steps of $10 \mathrm{~dB}$, as well as the clean ECG.

2.4. MSE Analysis for the Real ECG. We verified the sensitivity of MSE to SNR in the real ECG plus different types of noise (i.e., BW, EM, MA, and PL) using a SNR within the range from $40 \mathrm{~dB}$ to $-20 \mathrm{~dB}$ in steps of $10 \mathrm{~dB}$, as well as the real ECG.

\section{Results}

Figure 3 shows the mean value and standard deviation of the MSE of four kinds of signals (i.e., HF noise, LF noise, the clean ECG, and PL noise). The MSE value of the HF noise decreased monotonically with scale factor increased. Both of the MSE values of the LF noise and clean ECG increased monotonically with scale factor increased. The MSE of PL interference showed fluctuation when it increased with scale factor increased.

Figure 4(a) shows the effect of signal length on MSE for the artificial ECGs, that is, the clean artificial ECG plus LF, PL, or HF noise, as well as the clean artificial ECG. Figure 4(b) gives examples of the MSE using a scale factor of 5. Similarly, Figure 4(c) gives examples of MSE using a scale factor of 10. The MSE of all types of the ECG had a slight change when the signal length was below $10 \mathrm{~s}$. However, the MSE kept a steady value when signal length was longer than $10 \mathrm{~s}$. 


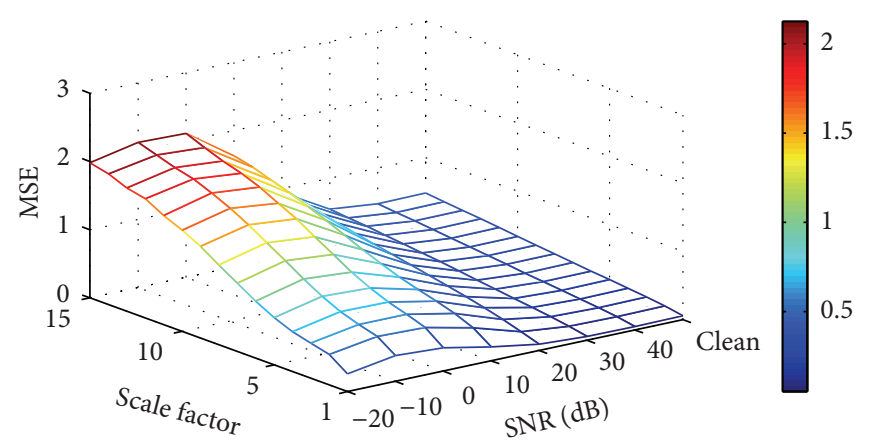

(a)

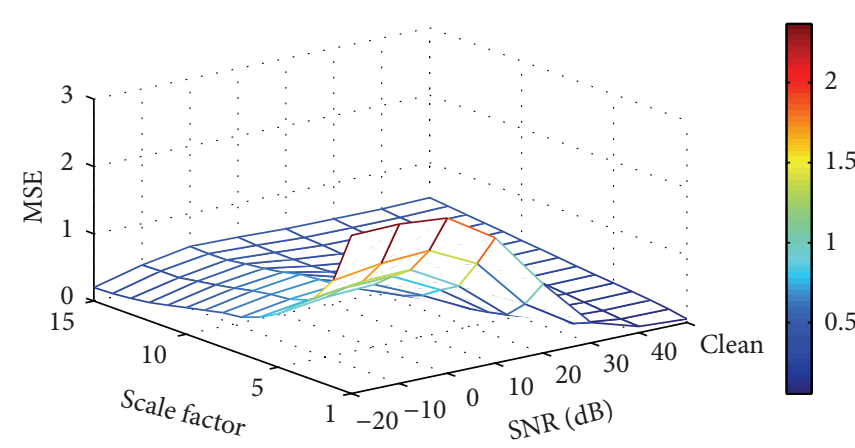

(c)

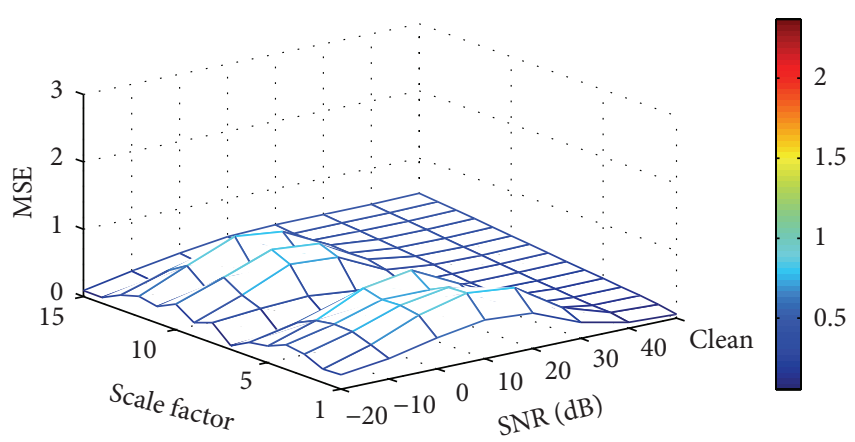

(e)

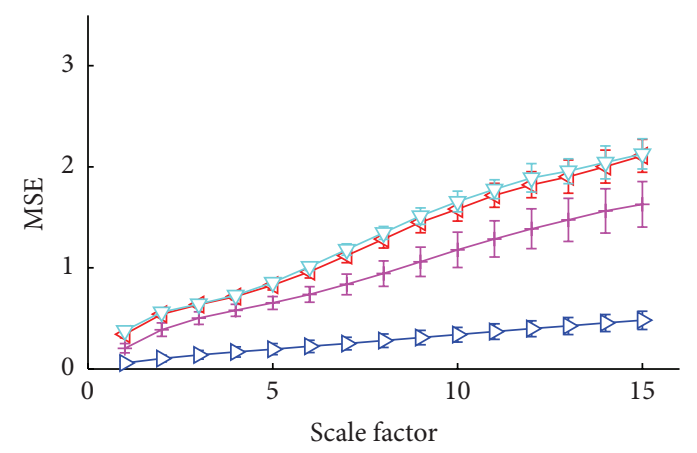

(b)

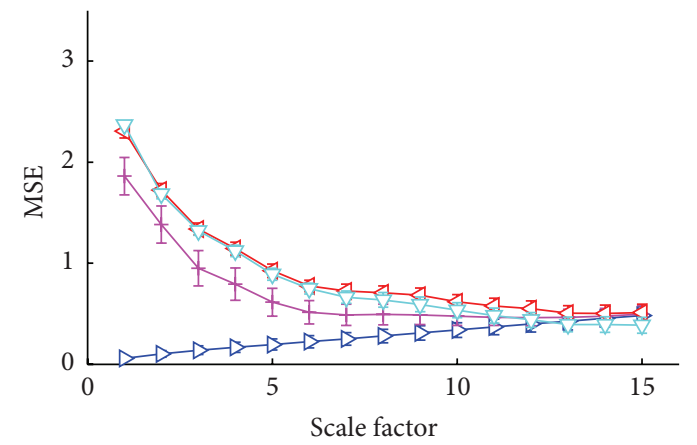

(d)

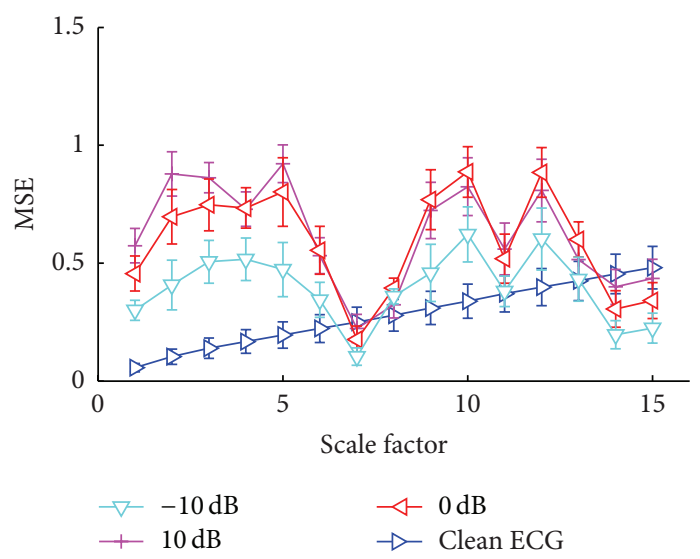

(f)

FIGURE 5: The results of SNR effect on the MSE for the artificial signals. (a) The 3D view of the relationship between MSE and SNRs of the clean ECG plus LF noise. (b) Examples of the clean ECG plus LF noise with SNRs of $-10 \mathrm{~dB}, 0 \mathrm{~dB}$, or $10 \mathrm{~dB}$, as well as the clean ECG. (c) The $3 \mathrm{D}$ view of the relationship between MSE and SNRs of the clean ECG plus HF. (d) Examples of the clean ECG plus HF with SNRs of -10 dB, $0 \mathrm{~dB}$, or $10 \mathrm{~dB}$, as well as the clean ECG. (e) The 3D view of the relationship between MSE and SNRs of the clean plus PL. (f) Examples of the clean plus PL noise with SNR of $-10 \mathrm{~dB}, 0 \mathrm{~dB}$, or $10 \mathrm{~dB}$, as well as the clean ECG.

Figure 5 shows the relationship between the SNR and the MSE for the artificial ECG signals. For the clean ECG plus LF noise and the clean ECG plus HF noise, the MSE increased with the decrease of the SNR when the SNR was larger than $-10 \mathrm{~dB}$ and then decreased when the SNR was less than $-10 \mathrm{~dB}$. For the clean ECG plus PL noise, the MSE showed fluctuation and increased with the decrease of the SNR when the SNR was larger than $10 \mathrm{~dB}$ and then decreased. Figure 5(a) shows that the MSE for the clean ECG plus LF noise increases relatively obviously within the higher range of scale factor, but Figure 5(c) shows that the MSE for the clean ECG plus HF noise significantly increases within the lower range of scale factor.

Figures 5(b), 5(d), and 5(f) show examples of the relationship between the SNR and the MSE for the different artificial ECG signals when the SNR is $-10 \mathrm{~dB}, 0 \mathrm{~dB}$, or $10 \mathrm{~dB}$, as well as the clean ECG.

Figure 6 shows the results of SNR affecting MSE for the real signals. For the real ECG plus BW, the MSE reached the maximum value when the SNR was $10 \mathrm{~dB}$ and then decreased 


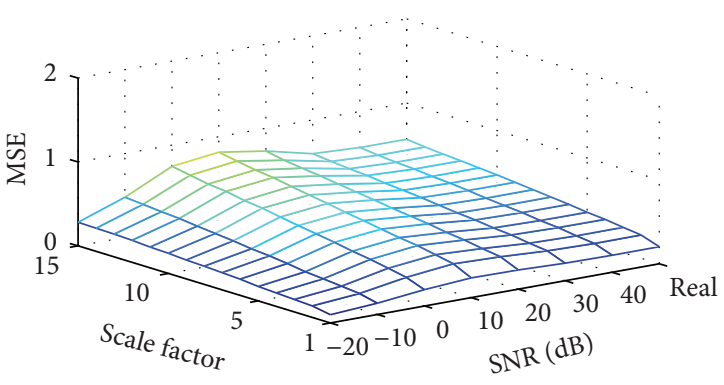

(a)

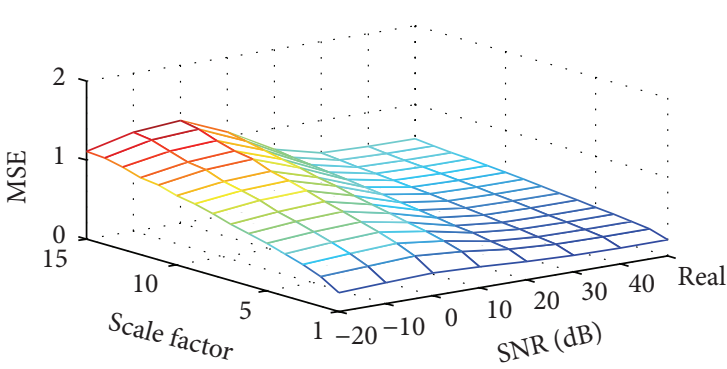

(c)

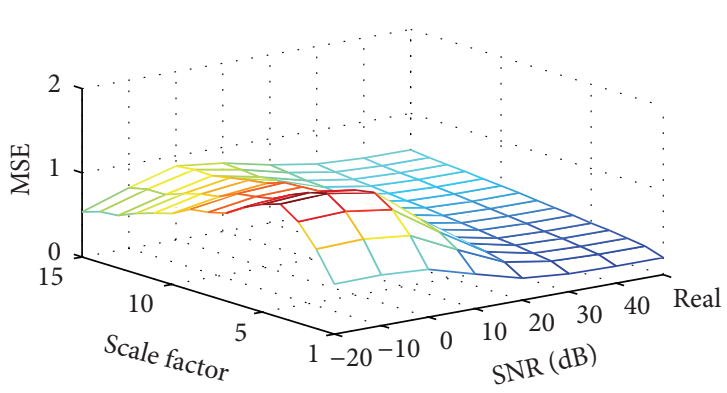

(e)

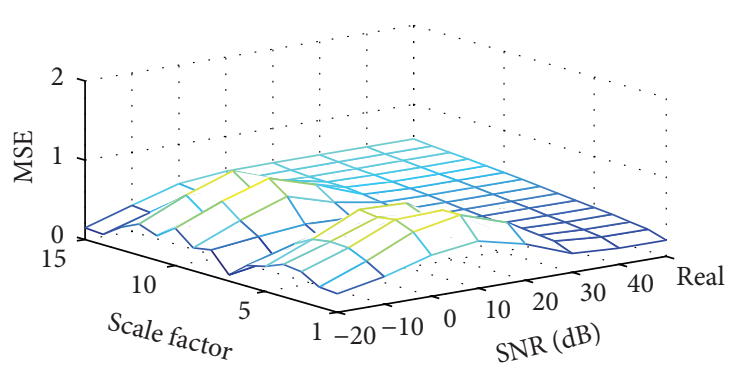

(g)

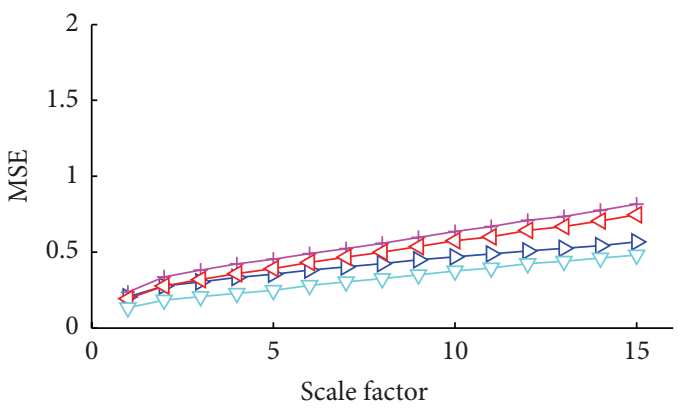

(b)

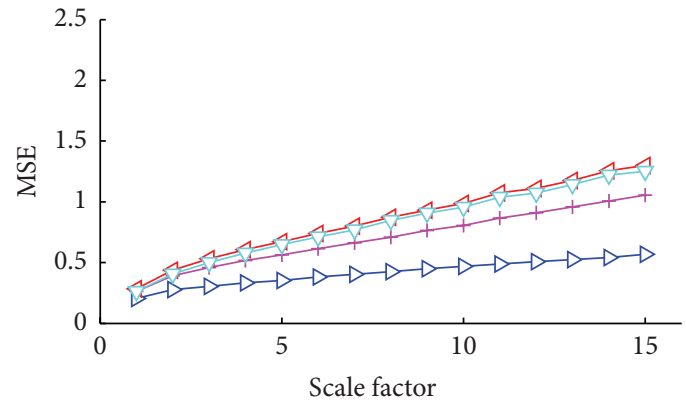

(d)

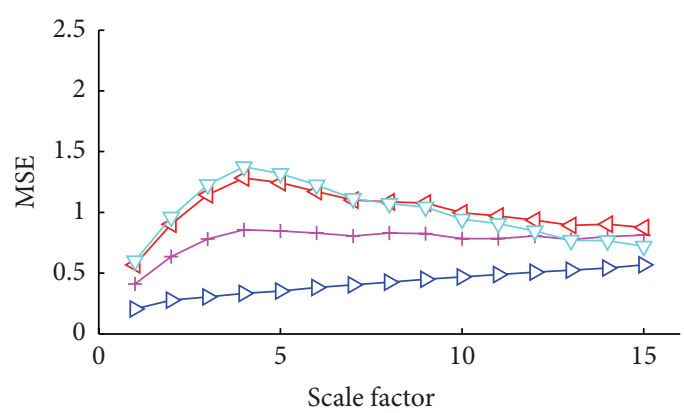

(f)

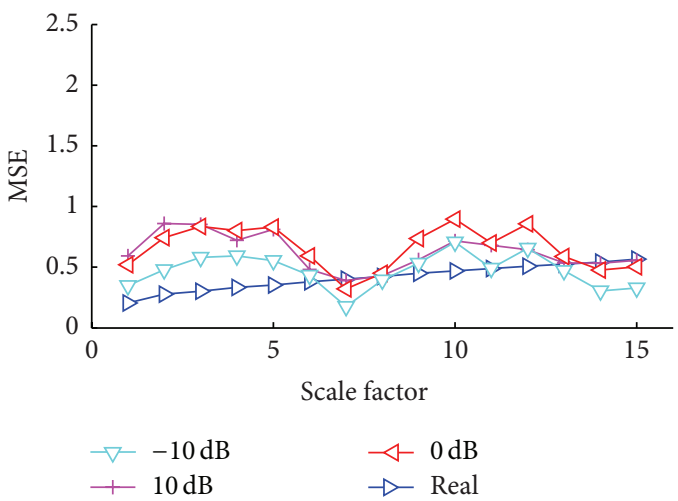

(h)

FIGURE 6: The validation results of SNR effect on MSE for the real ECG signals. (a), (c), (e), and (g) are the 3D view of the MSE of the real ECG plus BW, EM, MA, and PL, with SNR from $-20 \mathrm{~dB}$ to $40 \mathrm{~dB}$ with a step of $10 \mathrm{~dB}$, respectively. (b), (d), (f), and (h) are the MSE values of the noisy ECGs with $-10 \mathrm{~dB}, 0 \mathrm{~dB}, 10 \mathrm{~dB}$, and the real ECG, respectively. 
with the decrease of the SNR. However, for the real ECG plus EM or MA, the MSE increased with the decrease of the SNR when the SNR is larger than $-10 \mathrm{~dB}$, and then the MSE decreased with the decrease of the SNR. The MSE showed obvious fluctuation and increased with the decrease of the SNR when the SNR was larger than $0 \mathrm{~dB}$ and then decreased.

\section{Discussions}

This study tries to provide interpretation of the MSE measure in the context of quality classification of ECG collected via mobile phone.

The results indicate that the MSE is closely related to complexity of time series, instead of the periodicity and randomicity of time series. Thus, the MSE for LF noise and the clean ECG (quasiperiodic signal) monotonously increase with the increase of scale factor, but the MSE for HF noise monotonously decreases with the increase of scale factor. In addition, the MSE for PL noise (periodic signals) shows fluctuation. The results further indicate that change trends of the MSE for various types of artificial ECG signals are clearly different, and it means that the MSE can be applied to distinguish different noises contained in biomedical signal.

Actually, the MSE for ECG is independent of signal length when the length is longer than $10 \mathrm{~s}$. This point is beneficial for applying MSE to mobile ECG because the length of ECG collected by mobile devices is of relatively short duration. It is noted that the MSE also provides the possibility of real-time analysis since a longer signal means that more time is required to compute its MSE.

The results also show that the MSE is sensitive to SNR; that is, the MSE can reflect the content level of noises contained in the artificial or real ECG signals. For the real ECG signals, BW represents primarily baseline wander, a lowfrequency signal usually caused by motion of the subject or the electrodes. So the LF noise represents mostly BW, and EM contains electrode motion artifact, with significant amounts of baseline wander $[18,19]$. In this study, we also use the LF noise to approximately simulate EM. The trend of MSE for the real ECG plus BW or EM is consistent with that of the clean artificial ECG plus LF noise except that the corresponding SNR is different when the MSE reaches the maximum value because the real ECG signals always contain considerable LF noise, but the clean artificial ECG signals do not.

MA contains primarily muscle noise, and HF noise represents MA $[18,19]$. The trend of MSE for the real ECG plus MA is consistent with that of the clean artificial ECG plus HF noise except that the MSE for the real ECG plus MA noise is relatively larger within high range of scale factor because MA from the NSTDB also contains other noises.

Actually, when content level of noise contained in time series reaches a certain peak value, complexity of time series begins to decrease; that is, structure of time series tends to the steady state. Thus, for all of the synthetic ECG added different types of noise, the MSE firstly increases with the decrease of the SNR and then decreases.

For all of the synthetic ECG signals, the MSE is sensitive to the content level of noise contained in the signals. However the change trend of the MSE for the synthetic ECG added various noises is different. Thus we propose that the MSE should be applied to analyse the signal that is obviously contaminated by a single variety of noise, and the content level of other types of noise is relatively less.

Besides, for the synthetic ECG plus BW or EM, MSE is more sensitive to the change of SNR when scale factor is within higher range. However, for the synthetic ECG plus MA, MSE is more sensitive to SNR within lower range of scale factor. Thus, a proper scale factor has to be chosen in order that MSE is better sensitive to SNR for all types of the synthetic ECGs. This study suggests that a proper scale factor should be selected from 5 to 10 .

\section{Conclusions}

This study verified the performances of MSE for the assessment of ECG signal quality. Our results showed that MSE was sensitive to the noise level within the ECG signals and could, therefore, represent a valuable tool for the assessment of ECG signal quality.

\section{Conflict of Interests}

The authors declare that there is no conflict of interests regarding this work.

\section{Acknowledgments}

This work was supported by the Young Scientists Fund of the National Natural Science Foundation of China under Grant no. 61201049, the Excellent Young Scientist Awarded Foundation of Shandong Province in China under Grant no. BS2013DX029, and the China Postdoctoral Science Foundation under Grant no. 2013M530323. The authors would like to thank the MIT-BIH Arrhythmia Database for providing the invaluable data used in their research.

\section{References}

[1] Q. Li and G. D. Clifford, "Signal quality and data fusion for false alarm reduction in the intensive care unit," Journal of Electrocardiology, vol. 45, no. 6, pp. 596-603, 2012.

[2] G. D. Clifford and G. B. Moody, "Signal quality in cardiorespiratory monitoring," Physiological Measurement, vol. 33, no. 9, article E01, 2012.

[3] G. B. Moody, Physionet/Computing in Cardiology Challenge, 2011, http://physionet.org/challenge/2011.

[4] C. Liu, P. Li, L. N. Zhao, Y. Jing, and L. P. Liu, "Evaluation method for heart failure using RR sequence normalized histogram," in Proceedings of the 38th Computing in Cardiology, pp. 305-308, IEEE, Hangzhou, China, September 2011.

[5] P. Langley, L. Y. Di Marco, S. King et al., "An algorithm for assessment of quality of ECGs acquired via mobile telephones," in Proceedings of the 38th Computing in Cardiology, pp. 281-284, Hangzhou, China, September 2011.

[6] S. Zaunseder, R. Huhle, and H. Malberg, "Assessing the usability of ECG by ensemble decision trees," in Proceedings of the 38th Computing in Cardiology (CinC '11), pp. 277-280, Hangzhou, China, September 2011. 
[7] G. D. Clifford, J. Behar, Q. Li, and I. Rezek, "Signal quality indices and data fusion for determining clinical acceptability of electrocardiograms," Physiological Measurement, vol. 33, no. 9, pp. 1419-1433, 2012.

[8] Y. Zhang, C. Liu, S. Wei, C. Wei, and F. Liu, "ECG quality assessment based on a kernel support vector machine and genetic algorithm with a feature matrix," Journal of Zhejiang University SCIENCE C, vol. 15, no. 7, pp. 564-573, 2014.

[9] M. Costa, A. L. Goldberger, and C.-K. Peng, "Multiscale entropy analysis of complex physiologic time series," Physical Review Letters, vol. 89, no. 6, pp. 0681021-0681024, 2002.

[10] M. Costa, A. L. Goldberger, and C. K. Peng, "Multiscale entropy analysis of biological signals," Physical Review E: Statistical, Nonlinear, and Soft Matter Physics, vol. 71, no. 2, Article ID 021906, 18 pages, 2005.

[11] C.-C. Chung, J.-H. Kang, R.-Y. Yuan et al., "Multiscale entropy analysis of electroencephalography during sleep in patients with parkinson disease," Clinical EEG \& Neuroscience, vol. 44, no. 3 , pp. 221-226, 2013.

[12] X. Zhang, X. Chen, P. E. Barkhaus, and P. Zhou, "Multiscale entropy analysis of different spontaneous motor unit discharge patterns," IEEE Journal of Biomedical and Health Informatics, vol. 17, no. 2, pp. 470-476, 2013.

[13] P. A. Varotsos, N. V. Sarlis, E. S. Skordas, and M. S. Lazaridou, "Natural entropy fluctuations discriminate similar-looking electric signals emitted from systems of different dynamics," Physical Review E-Statistical, Nonlinear, and Soft Matter Physics, vol. 71, no. 1, Article ID 011110, 2005.

[14] P. E. McSharry, G. D. Clifford, L. Tarassenko, and L. A. Smith, "A dynamical model for generating synthetic electrocardiogram signals," IEEE Transactions on Biomedical Engineering, vol. 50, no. 3, pp. 289-294, 2003.

[15] G. B. Moody and R. G. Mark, "The impact of the MIT-BIH arrhythmia database," IEEE Engineering in Medicine and Biology Magazine, vol. 20, no. 3, pp. 45-50, 2001.

[16] A. L. Goldberger, L. A. Amaral, L. Glass et al., "PhysioBank, PhysioToolkit, and PhysioNet: components of a new research resource for complex physiologic signals," Circulation, vol. 101, no. 23, pp. e215-e220, 2000.

[17] G. B. Moody, W. E. Muldrow, and R. G. Mark, "A noise stress test for arrhythmia detectors," in Proceedings of the 11th Computers in Cardiology Conference, pp. 381-384, Park City, Utah, USA, 1984.

[18] M. Altuve, O. Casanova, S. Wong, G. Passariello, A. Hernandez, and G. Carrault, "Evaluación de dos Métodos para la Segmentación del Ancho de la Onda T en el ECG," in Proceedings of the 4th Latin American Congress on Biomedical Engineering Bioengineering Solutions for Latin America Health, pp. 12541258, Margarita Island, Venezuela, 2008.

[19] G. B. Moody and R. G. Mark, "The MIT-BIH Arrhythmia Database on CD-ROM and software for use with it," in Proceedings of the 17th Computers in Cardiology, pp. 185-188, Chicago, Ill, USA, September 1990. 

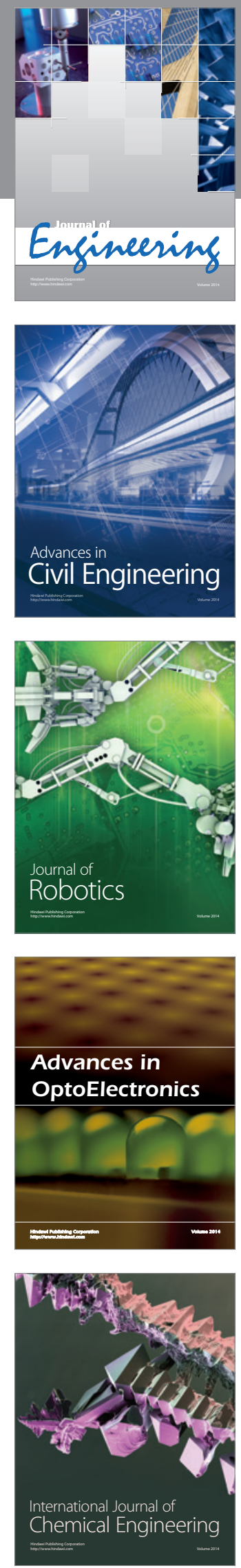

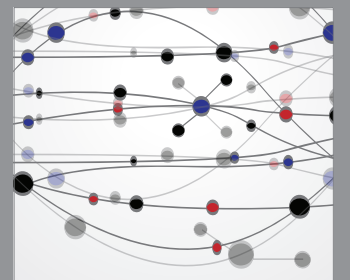

The Scientific World Journal
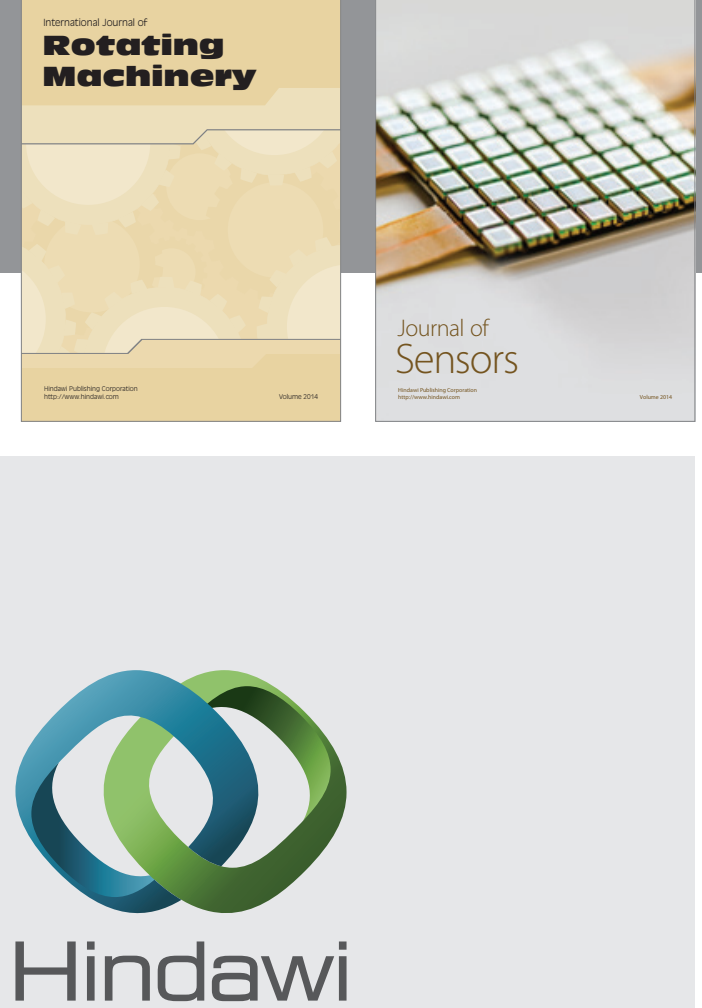

Submit your manuscripts at http://www.hindawi.com
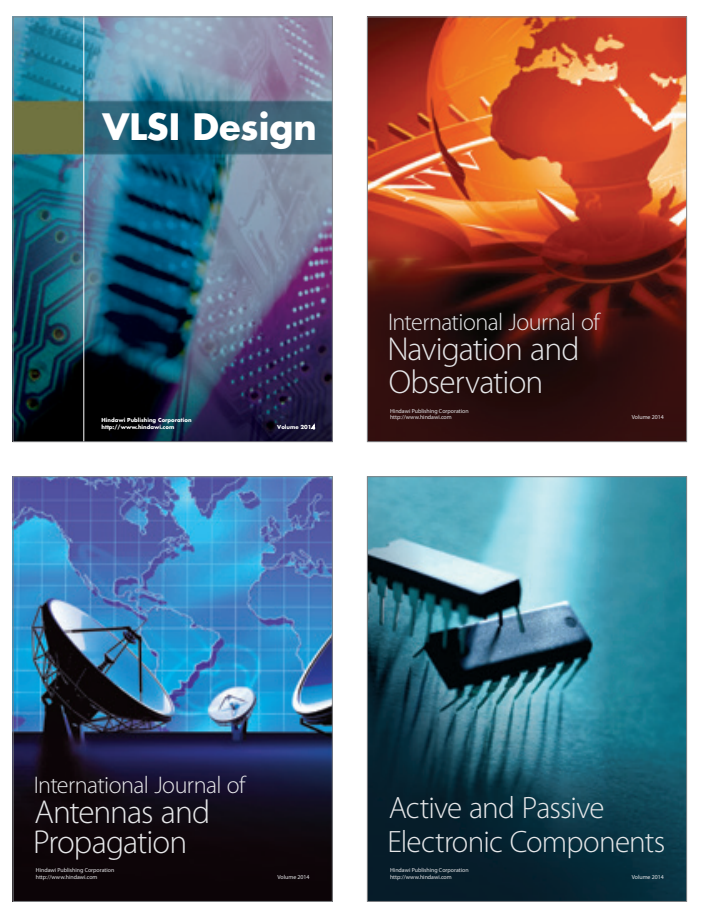
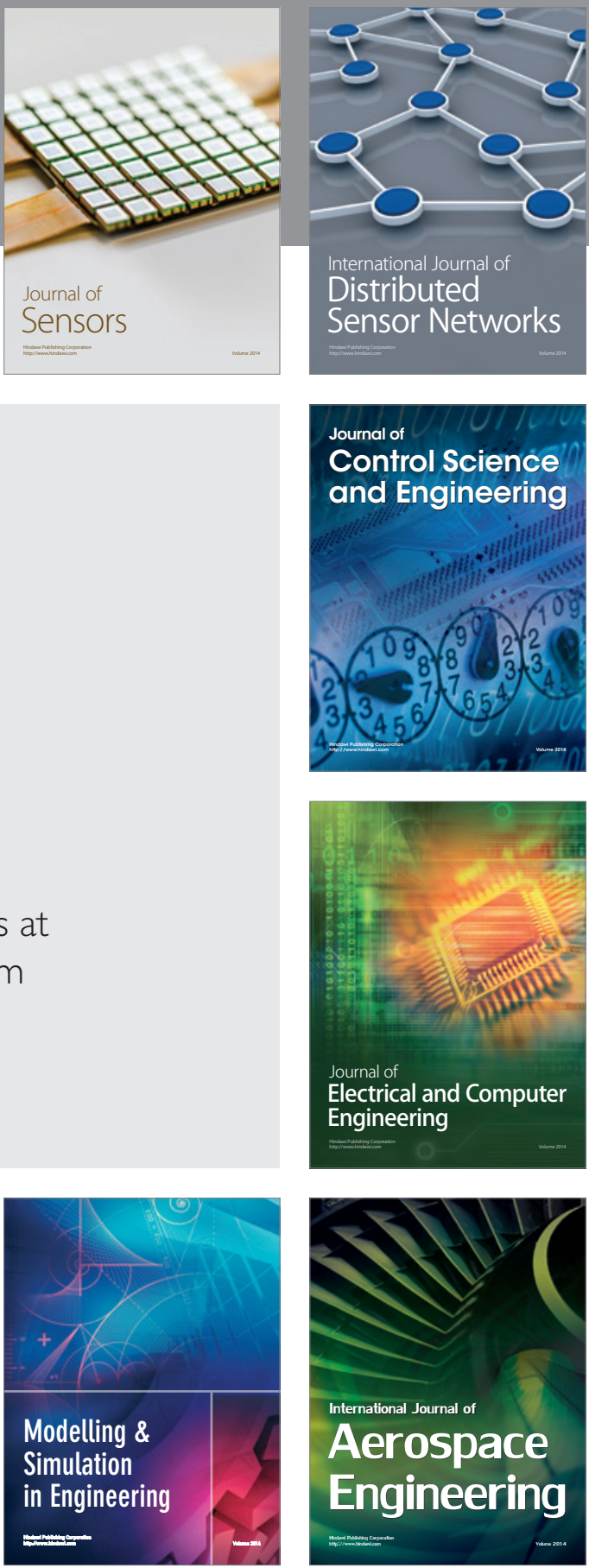

Journal of

Control Science

and Engineering
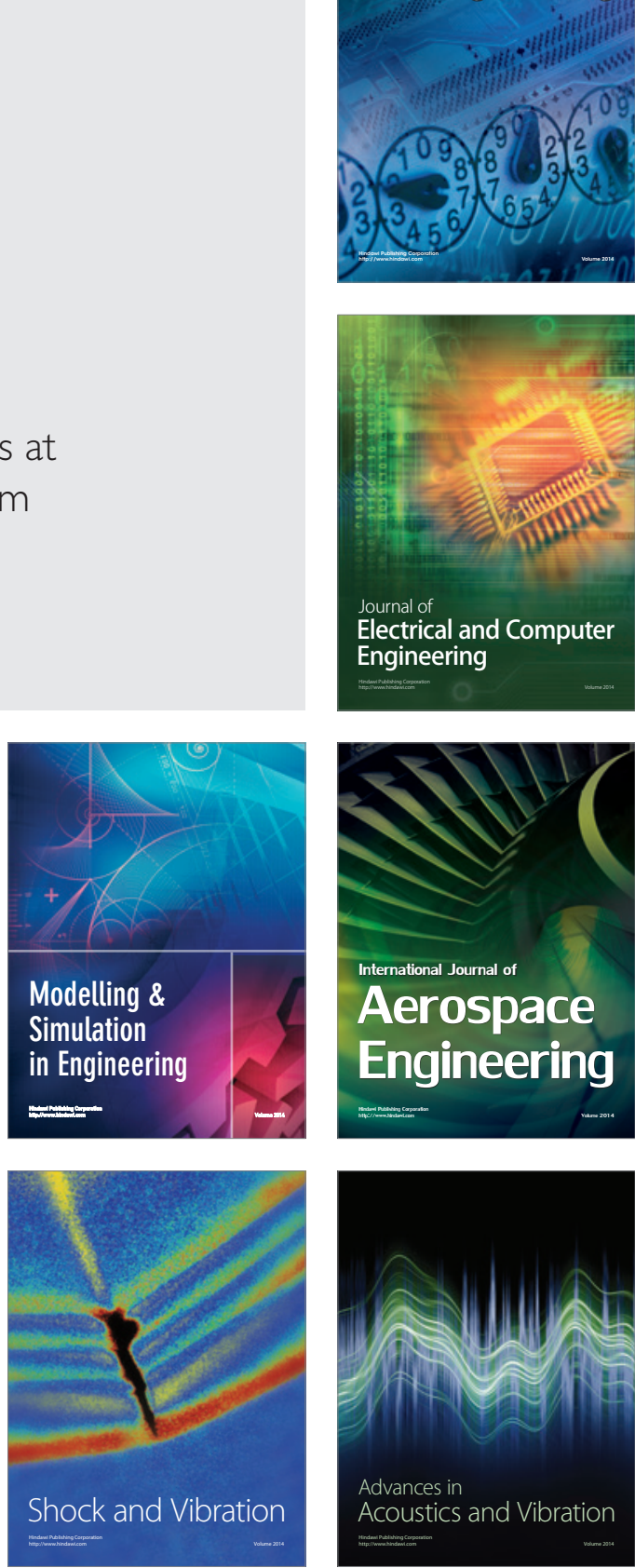